УДК 550.360; 550.832.6

\title{
ОЦЕНКА ТЕМПЕРАТУРНОГО ШУМА СВОБОДНОЙ ТЕПЛОВОЙ КОНВЕКЦИИ В ВОДОНАПОЛНЕННЫХ БУРОВЫХ СКВАЖИНАХ ПО ЭКСПЕРИМЕНТАЛЬНЫМ ДАННЫМ
}

\author{
Демежко Дмитрий Юрьевич1, \\ ddem54@inbox.ru
}

\author{
Хацкевич Богдан Дмитриевич 1 , \\ disaybl@yandex.ru
}

\author{
Миндубаев Мансур Габдрахимович1, \\ mansur_mg@mail.ru \\ 1 Институт геофизики УрО РАН, \\ Россия, 620016, г. Екатеринбург, ул. Амундсена, 100.
}

\begin{abstract}
Актуальность. Температурные исследования в скважинах используются для решения широкого круга разведочных, промыслово-геофизических, экологических, гидрогеологических, геодинамических задач. Появление новых температурных датчиков и систем регистрации существенно расширяет возможности скважинной термометрии, одновременно предъявляя повышенные требования к точности измерений, которые часто не могут быть удовлетворены в реальных скважинных условиях вследствие влияния температурного шума, вызываемого свободной тепловой конвекцией жидкости. Эффрективное планирование аппаратуры и методик температурных измерений в скважинах требует оценки амплитуды температурного шума.

Цель: обоснование математических моделей, позволяющих оценивать уровень температурного шума, вызываемого свободной тепловой конвекцией.

Методы: статистический анализ данных, полученных с помощью лабораторных экспериментов, в ходе температурного каротажа и мониторинга температуры в реальных скважинах.

Результаты. Обоснован оптимальный параметр оценки уровня температурного шума - среднеквадратическое отклонение температурных колебаний. Разработаны модели различной сложности, позволяющие оценивать амплитуду конвективного шума в зависимости от геотермического градиента, внутреннего радиуса скважины и числа Рэлея. Предложенные модели позволяют более эффрективно обосновать выбор аппаратуры и методики температурного каротажа и температурного мониторинга скважин в зависимости от соотношения «полезный сигнал/температурный шум».
\end{abstract}

\section{Ключевые слова:}

Скважина, температурный каротаж, температурный мониторинг, свободная тепловая конвекция, температурный шум.

\section{Введение}

При проведении прецизионных температурных измерений в скважинах исследователи часто сталкиваются с серьезной помехой - температурным шумом, вызываемым свободной тепловой конвекцией скважинного флюида. Конвективный температурный шум сопровождает как традиционные исследования естественного температурного поля [1-7], так и методы активной термометрии с использованием греющего кабеля [8-10]. Оценить величину температурного шума до проведения температурных измерений - важная научная задача, решение которой позволяет более эффективно планировать геотермические исследования.

Попытки связать амплитуду конвективных температурных колебаний с параметрами, определяющими свободную тепловую конвекцию, предпринимались и ранее Taк, W. Diment [11] оценил соотношение между максимальным диапазоном температурных возмущений и геотермическим градиентом $G$ в водонаполненной скважине диаметром $25 \mathrm{~cm}(r=0,125 \mathrm{м}): \Delta T_{\max }=1,25 G$ E. Sammel [12] опубликовал данные наблюдений размаха температурных колебаний и градиента в водонаполненных скважинах диаметром 4,8-10,2 см. Их линейная аппроксимация приводит к зависимости: $\Delta T_{\max }=0,31 G$ Более общая зависимость: $\Delta T_{\max }=A G r$, в которой безразмерная константа $A$ определена как отношение длины конвективной ячейки к радиусу скважины, была пред- ставлена в [1, 13]. L. Eppelbaum и I. Kutasov [6] предложили принципиально иную зависимость $\Delta T_{\max }=G /(D(1-B \lg R a))$, где $D$ и $B$ - константы, определяемые видом скважинного флюида. Для воды $D=6,25 \mathrm{~m}^{-1}, B=0,16$. Впрочем, из статьи $\lceil 6\rceil$ не ясно, как была получена эта формула и по каким экспериментальным данным она верифицировалась. При фиксированном значении температурного градиента с ростом $R a$ температурный шум растет, но при $R a \approx 1,8 \cdot 10^{6}$ резко уменьшается и становится отрицательным (?). Вряд ли такое поведение может быть физически обосновано.

\section{Критерии оценки амплитуды температурного шума}

Отметим, что все перечисленные выше зависимости предлагают оценивать амплитуду температурных колебаний их максимальным размахом. Это логично, если предположить, что конвективные течения организованы в виде вертикальной последовательности замкнутых конвективных ячеек, - долгие годы так и считалось. Тогда максимальный размах колебаний будет определяться разностью температур на нижней и верхней границах ячейки и при постоянном градиенте зависеть от ее вертикального размера.

В недавних исследованиях на основании численного математического моделирования [14-17] и лабораторных экспериментов [18] было показано, что конвективные потоки в вертикальном канале пред- 
ставляют собой вращающуюся систему винтовых струй и не ограничены по вертикали. Хотя обе статистики - и размах и среднеквадратическое отклонение - являются мерами рассеяния значений случайной величины, размах используется для оценки по небольшим выборкам и в случае если распределение случайной величины имеет естественные физические ограничения. Эта статистика крайне неустойчива к появлению больших выбросов. При отсутствии вертикального ограничения конвективных ячеек распределение температурных отклонений от среднего подчинено нормальному закону (рис. 1) и существует вероятность появления больших выбросов. В этих условиях естественной статистикой является среднеквадратическое отклонение. Для оценки среднеквадратического отклонения нами в [16] была предложена зависимость $\sigma \approx 3 G r$. Соотношение между максимальным размахом и среднеквадратическим отклонением можно примерно определить как $\Delta T_{\max }= \pm 2 \sigma=4 \sigma$ (с доверительной вероятностью 0,95 ).

В настоящей статье приведены результаты верификации этой зависимости $\sigma \approx 3 G r$ на большом объеме экспериментальных данных, полученных в лаборатории и in situ. Зависимость прослежена в пределах четырех порядков изменения градиента.

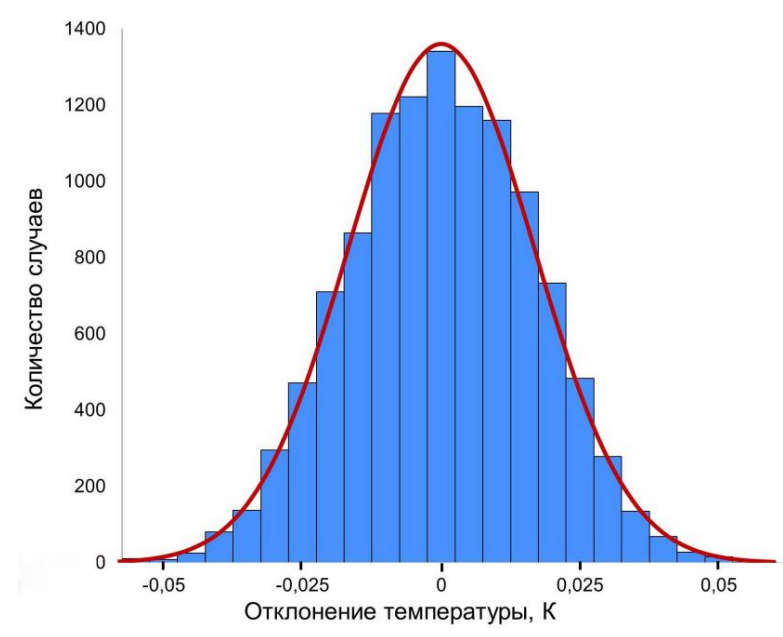

Рис. 1. Распределение отклонений температуры от среднего значения в скважине kun-1 на глубине 80 м (гистограмма) и теоретическое нормальное распределение (красная кривая). Гистограмма построена по данным 11424 отсчетов с интервалом дискретизачии 30 мин.

Fig. 1. Distribution of temperature deviations from the average value in the kun-1 borehole at a depth of 80 $m$ (histogram) and theoretical Gaussian distribution (red curve). The histogram is plotted using 11424 samples with a sampling interval of 30 minutes

\section{Данные}

В нашем анализе использован 171 ряд наблюдений температуры. Температуры были измерены в лабораторных условиях, а также в результате температурного мониторинга и температурного каротажа буровых скважин. Обобщенные исходные данные представлены в табл. 1.
Лабораторные оценки температурного шума (LabIRT-2, Lab-IRT-2) были получены при исследовании структуры конвективных течений методом инфракрасной термографии [18]. Скважина имитировалась вертикальными водонаполненными керамическими трубками с внутренним диаметром 20 и 30 мм. Геотермический градиент на внешних стенках трубы обеспечивал восходящий поток теплого воздуха от тороидального нагревателя. Температурные колебания регистрировались установленными у внутренней стенки трубы термопарами, подключенными к автоматическому регистратору.

В ходе температурного мониторинга в реальных скважинах нами были получены оценки по скважинам kun-1 на о-ве Кунашир (частично опубликованы в [7]), IGF-60 на геотермическом полигоне ИГФ УрО РАН в Екатеринбурге и К-1 (Катав-Ивановск, Челябинская обл.). Другие опубликованные мониторинговые данные - по скважине TG-2 (префектура Iwate, Япония [19]) и IODP-642E (подводная скважина в Норвежском море [20]). Все остальные данные были получены с помощью температурного каротажа (табл. 1).

Все данные, представленные в табл. 1, получены в условиях, когда число Рэлея, определяющее возникновение свободной тепловой конвекции, превышает критическое значение $\left(R a>R a_{\text {crit }}\right)$. Критическое число Рэлея для скважин меняется в пределах 68-216 в зависимости от соотношения теплопроводностей заполняющей скважину жидкости и окружающего ее массива $\lambda_{\mathrm{f}} / \lambda_{\mathrm{m}}$ [23]. Для необсаженной скважины (флюид-вода, $\lambda_{\mathrm{f}}=0,6 \mathrm{BT} \cdot \mathrm{M}^{-1} \cdot \mathrm{K}^{-1}$ при $20{ }^{\circ} \mathrm{C}$, внешний массив - горные породы, $\lambda_{\mathrm{m}}=2,5 \quad \mathrm{BT} \cdot \mathrm{M}^{-1} \cdot \mathrm{K}^{-1}$ ) $R a_{\text {crit }}=154$, для обсаженной (стальная обсадная труба, $\left.\lambda_{\mathrm{m}}=74 \mathrm{BT}^{\cdot} \mathrm{M}^{-1} \cdot \mathrm{K}^{-1}\right) R a_{\text {crit }}=212$.

Для двух ледниковых скважин (Гренландской GISP2_D и Антарктической WAIS_D) рассчитать число Рэлея не удалось. В качестве скважинного флюида в них использовались незамерзающие жидкости, теплофизические свойства которых неизвестны. Но можно предположить, что температурный шум в этих скважинах также связан со свободной тепловой конвекцией, поскольку иные его причины (вынужденная конвекция, аппаратурный шум) были исключены.

\section{Модели температурного шума}

Среднеквадратические отклонения температур рассчитывались после удаления пространственных (каротаж) или временных (мониторинг) трендов. Экспериментальная зависимость $\sigma r \approx k G$ приведена на рис. 2 .

Большая часть точек лежит в диапазоне $\sigma / r=(6 \div 1,5) G$. Линейная регрессия наименьших квадратов дает значение коэффициента $k=2,6$, близкое к теоретическому $(k=3,0)$. В то же время разброс оценок относительно теоретического значения весьма значителен. Рассмотрим возможные причины этого. Увеличение коэффициента $k>>3$ можно объяснить заниженной оценкой температурного градиента. Уменьшение температурного градиента в скважине в сравнении с невозмущенным в породах может наблюдаться в неглубоких скважинах или вблизи верхней или нижней границ [16]. В скважине IGF-60, внутренний радиус которой 52,5 мм, положительный 
температурный градиент, достаточный для инициации свободной тепловой конвекции, наблюдается в обводненной ее части на глубинах 7-10 м в конце мая-июне вследствие проникновения годовой температурной волны. При среднем значении градиента $G=0,034 \mathrm{~K} / \mathrm{м}$ здесь наблюдаются температурные колебания с амплитудой $\sigma=29-51$ мК (рис. 3). Это явно завышенная оценка. При подавлении конвекции вертикальными полиэтиленовыми пластинами [24] амплитуда колебаний уменьшается до 2-6 мК, а градиент увеличивается до ненарушенного значения $G=0,284$. Красные точки на рис. 3 построены для исходной амплитуды и ненарушенного градиента и хорошо согласуются с теоретической зависимостью.

таблица 1. Экспериментальные данные

Table 1. Experimental data

\begin{tabular}{|c|c|c|c|c|c|c|c|c|c|}
\hline 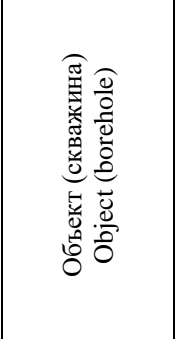 & $\begin{array}{l}\text { Расположение } \\
\text { Location }\end{array}$ & 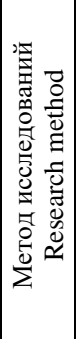 & 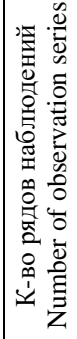 & 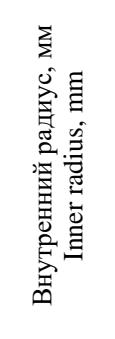 & 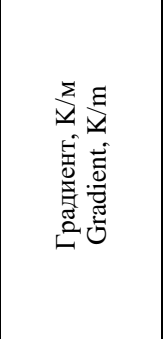 & $\sigma, \mathrm{mK}$ & $R a$ & 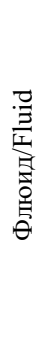 & $\begin{array}{l}\text { Примечания } \\
\text { ссылки } \\
\text { Notes } \\
\text { references }\end{array}$ \\
\hline Lab-IRT-2 & $\begin{array}{c}\text { ИГФ УрО РАН, Екатеринбург } \\
\text { IGF UB RAS } \\
\text { Yekaterinburg }\end{array}$ & $\begin{array}{l}\mathrm{M} \\
\mathrm{m}\end{array}$ & 54 & 10 & $1,56-11,89$ & $76-328$ & $3 \cdot 10^{3}-3 \cdot 10^{4}$ & $\begin{array}{l}\text { B } \\
\mathrm{W}\end{array}$ & $\begin{array}{c}\text { Данные } \\
\text { лабораторного } \\
\text { эксперимента }\end{array}$ \\
\hline Lab-IRT-2 & $\begin{array}{c}\text { ИГФ УрО РАН, Екатеринбург } \\
\text { IGF UB RAS } \\
\text { Yekaterinburg } \\
\end{array}$ & $\begin{array}{l}\mathrm{M} \\
\mathrm{m}\end{array}$ & 62 & 15 & $1,12-9,45$ & $86-320$ & $1 \cdot 10^{3}-1 \cdot 10^{4}$ & $\begin{array}{l}\text { B } \\
\mathrm{W}\end{array}$ & $\begin{array}{l}\text { Lab experiment } \\
\text { data }[18]\end{array}$ \\
\hline kun-1 & $\begin{array}{c}\text { О-в Кунашир, Сахалинская обл. } \\
\text { Kunashir Isl., Sakhalin Region } \\
\end{array}$ & $\begin{array}{l}\mathrm{M} \\
\mathrm{m}\end{array}$ & 7 & $52-84$ & $0,068-0,095$ & $8,0-16,8$ & $8,8 \cdot 10^{3}-6,8 \cdot 10^{4}$ & $\begin{array}{c}\mathrm{B} \\
\mathrm{W}\end{array}$ & [7] \\
\hline IGF-60 & \begin{tabular}{|c|} 
Геотерм. полигон ИГФ, \\
Екатеринбург \\
Geotherm. Observatory, IGF, \\
Yekaterinburg
\end{tabular} & $\begin{array}{l}\mathrm{M} \\
\mathrm{m}\end{array}$ & 4 & 53 & 0,284 & $28,8-50,6$ & $9 \cdot 10^{2}-1,7 \cdot 10^{3}$ & $\begin{array}{l}\mathrm{B} \\
\mathrm{W}\end{array}$ & \\
\hline $\mathrm{K}-1$ & $\begin{array}{c}\text { Катав-Ивановск, Челябинская обл. } \\
\text { Katav-Ivanovsk, Chelyabinsk } \\
\text { Region }\end{array}$ & $\begin{array}{l}\mathrm{M} \\
\mathrm{m}\end{array}$ & 3 & 150 & 0,010 & $3,5-8,7$ & $1,0 \cdot 10^{4}$ & $\begin{array}{l}\text { B } \\
\mathrm{W}\end{array}$ & \\
\hline TG-2 & $\begin{array}{c}\text { Преф. Ивате, Япония } \\
\text { Iwate Pref., Japan } \\
\end{array}$ & $\begin{array}{l}\mathrm{M} \\
\mathrm{m}\end{array}$ & 5 & 170 & 0,213 & $23,3-30,4$ & $6,7 \cdot 10^{6}$ & $\begin{array}{l}\mathrm{B} \\
\mathrm{W}\end{array}$ & [19] \\
\hline WAIS_D & $\begin{array}{c}\text { Taylor Dome, Антарктида } \\
\text { Antarctica }\end{array}$ & $\begin{array}{c}\mathrm{K} \\
1 \\
\end{array}$ & 6 & 81,5 & $0,006-0,025$ & $1,0-4,5$ & & $\begin{array}{l}\text { и } \\
\text { i }\end{array}$ & [21] \\
\hline GISP2_D & $\begin{array}{c}\text { Гренландия } \\
\text { Greenland } \\
\end{array}$ & $\begin{array}{l}\kappa \\
1\end{array}$ & 2 & 90,5 & $0,06-0,024$ & $1,3-6,2$ & & $\begin{array}{l}\mathrm{H} \\
\mathrm{n}\end{array}$ & {$[22]$} \\
\hline Hausen & $\begin{array}{c}\text { Северная Швейцария } \\
\text { Northern Switzerland }\end{array}$ & $\begin{array}{l}\kappa \\
1 \\
\end{array}$ & 3 & 87,5 & 0,045 & $6,1-10,0$ & $3,5 \cdot 10^{4}$ & $\begin{array}{l}\mathrm{B} \\
\mathrm{W}\end{array}$ & {$[1]$} \\
\hline IODP-1165C & $\begin{array}{c}\text { Prydz Bay, Антарктика } \\
\text { Antarctica } \\
\end{array}$ & $\begin{array}{l}\kappa \\
1\end{array}$ & 2 & $178-191$ & $0,015-0,043$ & $8,8-11,1$ & $1,6 \cdot 10^{5}-8,6 \cdot 10^{5}$ & $\begin{array}{c}\mathrm{B} \\
\mathrm{W}\end{array}$ & \multirow{10}{*}{$\begin{array}{c}\text { Подводные сква- } \\
\text { жины, пробурен- } \\
\text { ные по программе } \\
\text { The International } \\
\text { Ocean Discovery } \\
\text { Program (IODP) } \\
\text { [20] } \\
\text { Boreholes drilled } \\
\text { according to the the } \\
\text { International Ocean } \\
\text { Discovery Program } \\
\text { (IODP) }\end{array}$} \\
\hline IODP-1166A & $\begin{array}{c}\text { Prydz Bay, Антарктика } \\
\text { Antarctica } \\
\end{array}$ & $\begin{array}{l}\mathrm{K} \\
1 \\
\end{array}$ & 1 & 165 & 0,009 & 15,5 & $3,9 \cdot 10^{3}$ & $\begin{array}{l}\mathrm{B} \\
\mathrm{W}\end{array}$ & \\
\hline IODP-1168A & $\begin{array}{c}\text { Западный склон Тасмании } \\
\text { West Tasmania Slope }\end{array}$ & $\begin{array}{l}\mathrm{K} \\
1\end{array}$ & 3 & $158-173$ & $0,012-0,064$ & $9,0-15,2$ & $1,2 \cdot 10^{5}-9,5 \cdot 10^{5}$ & $\begin{array}{l}\mathrm{B} \\
\mathrm{W}\end{array}$ & \\
\hline IODP-1179D & \begin{tabular}{|c|} 
Возвыш. Шатского, \\
с-з Тихого океана \\
Shatsky Rise, NW Pacific \\
\end{tabular} & $\begin{array}{l}\kappa \\
1\end{array}$ & 1 & 211 & 0,013 & 12,8 & $3,4 \cdot 10^{4}$ & $\begin{array}{l}\text { B } \\
\mathrm{W}\end{array}$ & \\
\hline IODP-U1309D & $\begin{array}{c}\text { Срединно-Атлантический хребет } \\
\text { Mid Atlantic Ridge }\end{array}$ & $\begin{array}{l}\mathrm{K} \\
1 \\
\end{array}$ & 4 & $127-170$ & $0,026-0,150$ & $10,9-37,4$ & $1,8 \cdot 10^{5}-1,2 \cdot 10^{6}$ & $\begin{array}{l}\mathrm{B} \\
\mathrm{W}\end{array}$ & \\
\hline IODP-642E & $\begin{array}{c}\text { Voring Plateau, Норвежское море } \\
\text { Norwegian Sea }\end{array}$ & $\begin{array}{l}\mathrm{KM} \\
1 \mathrm{~m} \\
\end{array}$ & 3 & $37-133$ & $0,010-1,110$ & $7,2-92,1$ & $2,7 \cdot 10^{4}-2,7 \cdot 10^{5}$ & $\begin{array}{l}\mathrm{B} \\
\mathrm{W} \\
\end{array}$ & \\
\hline IODP-U1362A & \begin{tabular}{|c|} 
Juan de Fuca Flank, \\
с-в Tихого океана \\
NE Рacific \\
\end{tabular} & $\begin{array}{l}\kappa \\
1\end{array}$ & 2 & $134-157$ & $0,033-0,256$ & $11,7-86,2$ & $1,0 \cdot 10^{5}-1,9 \cdot 10^{5}$ & $\begin{array}{l}\text { B } \\
\mathrm{W}\end{array}$ & \\
\hline IODP-U1256D & $\begin{array}{c}\text { Guatemala Basin, экваториальн. } \\
\text { часть Тихого океана } \\
\text { Equatorial Pacific } \\
\end{array}$ & $\begin{array}{l}\kappa \\
1\end{array}$ & 3 & $140-171$ & $0,020-0,597$ & $17,4-94,9$ & $4,1 \cdot 10^{5}-2,2 \cdot 10^{7}$ & $\begin{array}{l}\text { B } \\
\mathrm{W}\end{array}$ & \\
\hline IODP-395A & $\begin{array}{c}\text { Срединно-Атлантический хре- } \\
\text { бет, центр. троп. } \\
\text { Mid Atlantic Ridge, Central } \\
\text { Tropical North Atlantic }\end{array}$ & $\begin{array}{l}\kappa \\
1\end{array}$ & 3 & 145 & $0,059-0,147$ & $31,4-78,9$ & $1,9 \cdot 10^{4}-6,5 \cdot 10^{5}$ & $\begin{array}{l}\text { B } \\
\mathrm{W}\end{array}$ & \\
\hline $\begin{array}{c}\text { IODP- } \\
\text { U1309D-2 }\end{array}$ & $\begin{array}{c}\text { Срединно-Атлантический хребет } \\
\text { Mid Atlantic Ridge }\end{array}$ & $\begin{array}{l}\kappa \\
1\end{array}$ & 2 & 156 & $0,083-0,116$ & $30,2-45,4$ & $9,7 \cdot 10^{5}-2,0 \cdot 10^{6}$ & $\begin{array}{l}\text { B } \\
\mathrm{W}\end{array}$ & \\
\hline
\end{tabular}

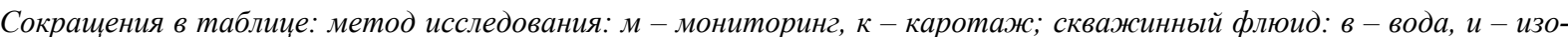
пар-к с утяжелителем, н-н-бутилацетат.

Abbreviations in the table: research method: $m$ - monitoring, $l$ - logging; borehole fluid: $w-w a t e r, i-i$ sopar- $k$ with densifying agent, $n-n$-butyl acetate. 


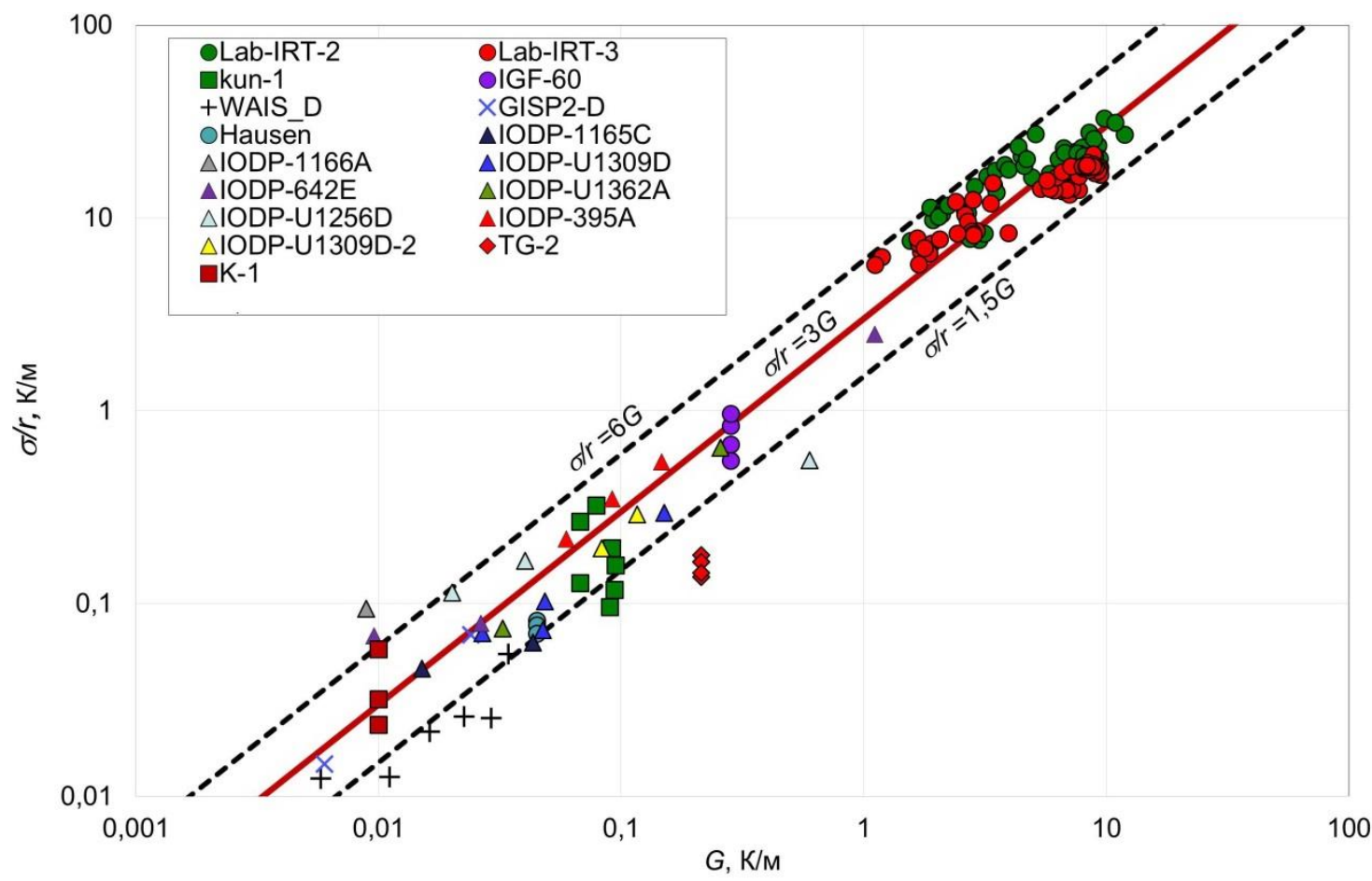

Pис. 2. Экспериментальная зависимость среднеквадратического отклонения температурного иума, нормированного на радиус скважины $r$, от геотермического градиента $G$. Прямые линии соответствуют соотношениям $\sigma / r=k G$ при различных значениях $k$

Fig. 2. Experimental dependence of the standard deviation of temperature noise normalized to the borehole radius $r$ from temperature gradient $G$. Straight lines correspond to the relations $\sigma / r=k G$ under different $k$ values

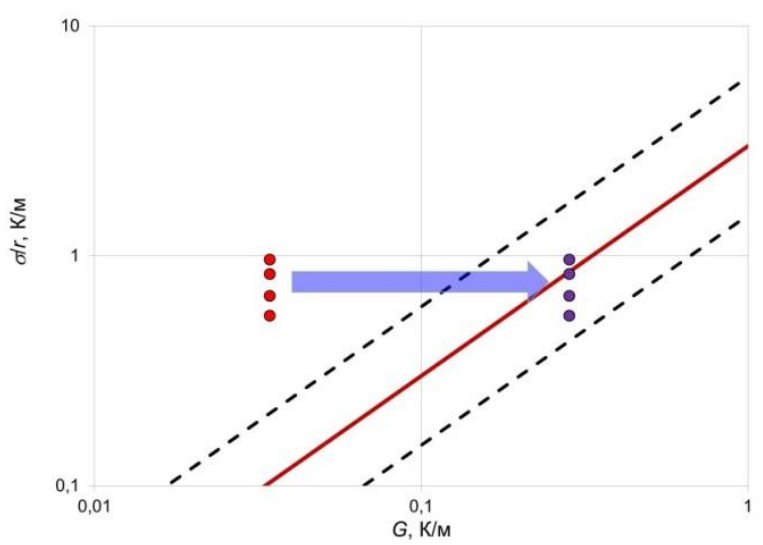

Рис. 3. Соотночение между среднеквадратическим отклонением температурного шума, нормированным на радиус скважины $r$, и температурным градиентом $G$ в скважине ИГФ-60. Красные точки соответствуют $G$, измеренному до подавления конвекиии, синие - после. Измерения проводились на глубинах 6,5-8,0 м (0,5-2,0 м ниже уровня грунтовых вод)

Fig. 3. Relationship between the standard deviation of temperature noise normalized to the borehole radius $r$ and the temperature gradient $G$ in the IGF-60 borehole. Red dots correspond to the temperature gradient which was measured before suppression of convection, blue ones - after suppression. The measurements were carried out at depths of 6,5-8,0 m (0,5-2,0 $\mathrm{m}$ below the groundwater level)

Уменьшение коэффициента до $k<<3$, в частности, можно объяснить влиянием системы температурных измерений, занимающей некоторую часть пространства скважины, т. е. уменьшающей ее эффективный радиус. Другое возможное объяснение - влияние инерционности системы измерений. В [16] было показано, что среднеквадратическое отклонение температурных колебаний практически не зависит от числа Рэлея. Однако при увеличении $R a$ спектральная плотность колебаний смещается в область высоких частот, которые отфильтровываются системой измерений. На рис. 4 приведена экспериментальная выборка в координатах $(\sigma / r ; G)$, в которой цветом обозначена величина числа Рэлея. Действительно, самые значительные отрицательные отклонения $\sigma / r$ от основной зависимости соответствуют самым большим числам Рэлея: $R a>1 \cdot 10^{6}$.

\section{Сравнение моделей температурного шума}

Рассмотрим несколько моделей температурного шума. Для характеристики качества модели используем средний модуль относительной ошибки:

$$
\delta=\frac{1}{n} \sum_{i=1}^{n} \frac{\left|\sigma_{\text {мод } i}-\sigma_{i}\right|}{\sigma_{i}},
$$

где $\sigma_{\text {мод }}$ и $\sigma$ - соответственно модельная и экспериментальная оценки среднеквадратического отклонения температурного шума; $n$ - объем выборки. В отличие от среднеквадратических, эта мера не преувеличивает влияния больших значений $\sigma$ и отдельных выбросов. Результаты сравнения трех моделей приведены в табл. 2. 


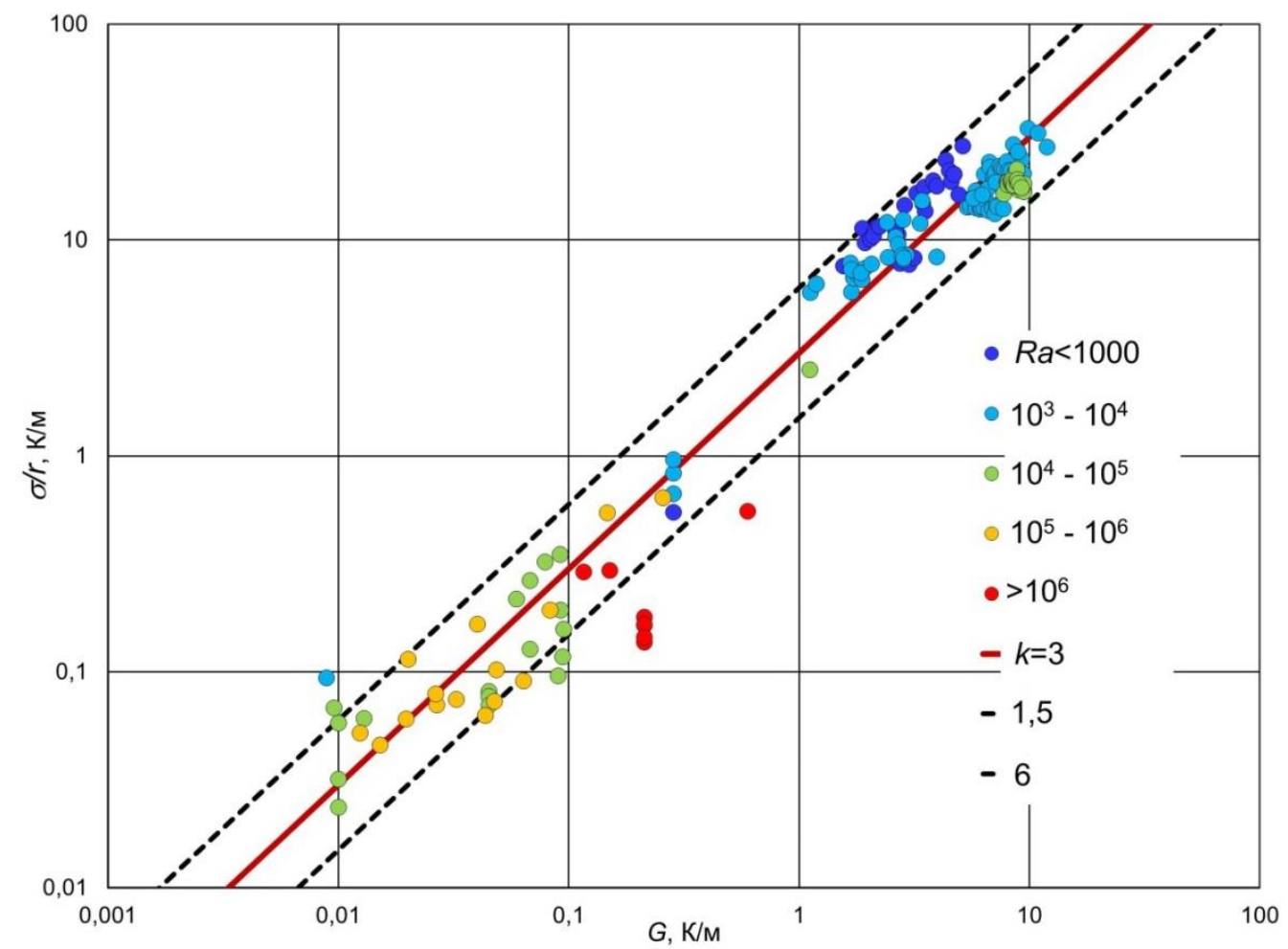

Pис. 4. Зависимость среднеквадратического отклонения температурного шума, нормированного на радиус скважины $r$, от геотермического градиента $G$ при различных значениях числа Рэлея (обозначено иветом). Прямые линии соответствуют соотношениям $\sigma / r=k G, k=1,5 ; 3 ; 6$

Fig. 4. Dependence of the standard deviation of temperature noise normalized to the borehole radius $r$ from temperature gradient $G$ for different values of Rayleigh number (indicated by color). Straight lines correspond to the relations $\sigma / r=k G, k=1,5 ; 3 ; 6$

Таблица 2. Значения среднего модуля относительной ошибки $\delta$ оченки конвективного шума бдля различных моделей

Table 2. Mean absolute percentage error $\delta$ of convective noise $\sigma$ forecast by different models

\begin{tabular}{|c|c|c|}
\hline № & Модель/Model & $\delta$ \\
\hline 1 & $\sigma_{\text {мод }}=2,3 \mathrm{Gr}$ & 0,34 \\
\hline 2 & $\sigma_{\text {мод }}=14,3 r \mathrm{G}^{0,89} / \mathrm{Ra}^{0,18}$ & 0,21 \\
\hline 3 & $\sigma_{\text {мод }}=\Delta T_{\max } / 4=G /(25(1-0,16 \lg R a))[6]$ & 1,55 \\
\hline
\end{tabular}

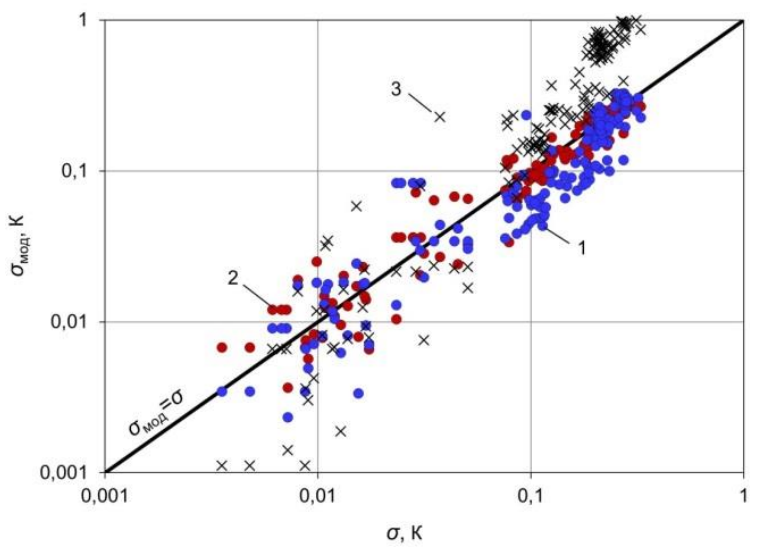

Рис. 5. Сравнение экспериментальных и модельных оценок амплитуды температурного шума. Номера на графике соответствуют номерам моделей в табл. 2

Fig. 5. Comparison of experimental and model estimates of the temperature noise amplitude. The numbers on the graph correspond to the model numbers in the table 2
Отметим, что минимум $\delta$ в простейшей модели достигается при $k=2,3$. Модель, учитывающая влияние числа Рэлея, дает наименьшую относительную ошибку прогноза, однако вряд ли ее можно рекомендовать в качестве универсальной модели. Как мы предположили выше, это влияние может быть обусловлено инерционностью конкретной системы измерений. Наибольшей относительной ошибкой характеризуется модель, предложенная в [6]. Эта модель переоценивает большие значения $\sigma$ и преуменьшает малые (рис. 5), а при $R a>1,8 \cdot 10^{6}$ оценка $\sigma$ становится вовсе отрицательной.

\section{Заключение}

Проведенные исследования позволили обосновать оптимальный параметр оценки уровня температурного шума, обусловленного свободной тепловой конвекцией в скважинах, - среднеквадратическое отклонение температурных колебаний. Предложенные модели оценки температурного шума базируются на большой выборке экспериментальных данных и позволяют более эффективно обосновать выбор аппаратуры и методики температурного каротажа (температурного мониторинга скважин) в зависимости от требуемого соотношения «полезный сигнал/температурный шум».

Исследование выполнено при поддержке РФФИ, проект № 19-05-00050-а (проведение полевых экспериментальных исследований в скважсинах, сбор и анализ данных температурного каротажа и мониторинга) и госбюджетной темьг НИР № 0394-2019-0002 (проведение исследований на лабораторной установке для моделирования свободной тепловой конвекции). 


\section{СПИСОК ЛИТЕРАТУРЫ}

1. Pfister M., Rybach L. High-resolution digital temperature logging in areas with significant convective heat transfer //Geothermics. 1995. - V. 24. - P. 99-100.

2. Field comparison of conventional and new temperature logging systems / K.W. Wisian, D.D. Blackwell, S. Bellani, J.A. Henfling, R.A. Norman, P.C. Lysne, A. Forster, J. Schrotter // Geothermics. - 1998. - V. 27. - P. 131-141.

3. Cermak V., Safanda J., Bodri L. Precise temperature monitoring in boreholes: Evidence for oscillatory convection? Part I Experiments and field data // International Journal of Earth Sciences - 2008. - V. 97 (2). - P. 365-373.

4. Cermak V., Safanda J., Bodri L. Thermal instability of the fluid column in a borehole: application to the Yaxcopoil hole (Mexico) // International Journal of Earth Sciences. - 2010. - V. 99 (6). P. 1437-1451.

5. Berthold S., Börner F. Detection of free vertical convection and double-diffusion in groundwater monitoring wells with geophysical borehole measurements // Environmental geology 2008. - V. 54 (7). - P. 1547-1566.

6. Eppelbaum L.V., Kutasov I.M. Estimation of the effect of thermal convection and casing on the temperature regime of boreholes: a review // Journal of Geophysics and Engineering. - 2011. V. 8 (1). - P. 1-10.

7. О природе температурных вариаций в скважине Kun-1 (о. Кунашир) / Д.Ю. Демежко, А.К. Юрков, В.И. Уткин, А.В. Климшин // Геология и геофизика. - 2012. - Т. 53. № 3. - C. 406-414.

8. Экспериментальная установка для изучения свободной тепловой конвекции при индукционном нагреве эксплуатационной колонны / Р.А. Валиуллин, Р.Ф. Шарафутдинов, В.Я. Федотов, И.В. Канафин // Вестник Башкирского университета. - 2016. T. 21. - № 2. - C. 264-268.

9. Distributed thermal response tests using a heating cable and fiber optic temperature sensing / M. Vélez Márquez, J. Raymond, D. Blessent, M. Philippe, N. Simon, O. Bour, L. Lamarche // Energies - 2018. - V. 11 (11). - P. 1-24.

10. Improved characterization of groundwater flow in heterogeneous aquifers using granular polyacrylamide (PAM) gel as temporary grout / M.V. Klepikova, C. Roques, S. Loew, J. Selker // Water Resources Research. - 2018. - V. 54 (2). - P. 1410-1419.

11. Diment W.H. Thermal regime of a large diameter borehole: instability of the water column and comparison of air- and waterfilled conditions // Geophysics. - 1967. - V. 32. - P. 720-726.

12. Sammel E.A. Convective flow and its effect on temperature logging in small-diameter wells // Geophysics. - 1968. V. 33 (6). - P. 1004-1012.
13. Diment W.H., Urban Th.C. A simple method for detecting anomalous fluid motions in boreholes from continuous temperature logs // GRC Trans. - 1983. - V. 7. - P. 485-490.

14. Миндубаев М.Г., Демежко Д.Ю. Свободная тепловая конвекция в буровых скважинах: численное моделирование и экспериментальные данные // Мониторинг. Наука и технологии. 2012. - № 4 (13). - C. 12-18.

15. Хорошев А.С. Численное исследование свободноконвективных течений в протяжённых вертикальных цилиндрических областях при постоянном вертикальном градиенте температуры на боковой поверхности // Вестник Самарского государственного аэрокосмического университета. - 2012. № 5-1 (36). - C. 46-48.

16. Демежко Д.Ю., Миндубаев М.Г., Хацкевич Б.Д. Температурные эффекты свободной тепловой конвекции в буровых скважинах // Геология и геофизика. - 2017. - Т. 58. - № 10. C. $1602-1610$.

17. Хорошев А.С., Шахов В.Г. Интенсивность конвекции жидкостей с разным числом Прандтля в вертикальном цилиндре большого удлинения // Математическая физика и компьютерное моделирование. - 2018. - Т. 21. - № 1. - С. 70-79.

18. Демежко Д.Ю., Хацкевич Б.Д., Миндубаев М.Г. Исследование свободной тепловой конвекции в вертикальном водонаполненном цилиндре методом инфракрасной термографии // Геология и геофизика. - 2019. - Т. 60. - № 7. - С. 1028-1035.

19. Long-term temperatures measurements at some onland and subseafloor boreholes / M. Kinoshita, K. Sayanagi, T. Kasaya, E. Araki, H. Ito // JAMSTEC Report of Research and Development. - 2009. - P. 89-102.

20. The International Ocean Discovery Program (IODP). URL: https://mlp.ldeo.columbia.edu/logdb/scientific_ocean_drilling/resu lt/ (дата обращения 15.08.2020).

21. Cuffey K.M., Clow G.D. Temperature profile of the West Antarctic ice sheet divide deep borehole // U.S. Antarctic Program (USAP) Data Center. - 2014. URL: http://www.usapdc.org/view/dataset/609550 (дата обращения 15.01.2020).

22. Clow G.D., Saltus R.W., Waddington E.D. A new high-precision borehole-temperature logging system used at GISP2, Greenland, and Taylor Dome, Antarctica // Journal of Glaciology. - 1996. V. 42 (142). - P. 576-584.

23. Гершуни Г.Э., Жуховиций Г.М. Конвективная устойчивость несжимаемой жидкости. - М.: Наука, 1972. - 393 с.

24. Способ температурного мониторинга в водонаполненных скважинах: пат. Рос. Федерация, № 26781746, заявл. 12.03.2018, опубл. 23.01.2019, Бюл. № 3. - 8 c.

Поступила 17.06.2020 2.

\section{Информация об авторах}

Демежко Д.Ю., доктор геолого-минералогических наук, главный научный сотрудник лаборатории геодинамики, Институт геофизики УрО РАН.

Хацкевич Б.Д., младший научный сотрудник лаборатории геодинамики, Институт геофизики УрО РАН.

Миндубаев М.Г., кандидат физико-математических наук, старший научный сотрудник лаборатории геодинамики, Институт геофизики УрО РАН. 
UDC 550.360; 550.832.6

\title{
EXPERIMENTAL ESTIMATION OF TEMPERATURE NOISE CAUSED BY FREE THERMAL CONVECTION IN WATER-FILLED BOREHOLES
}

\author{
Dmitry Yu. Demezhko1, \\ ddem54@inbox.ru
}

\author{
Bogdan D. Khatskevich¹, \\ disaybl@yandex.ru \\ Mansur G. Mindubaev ${ }^{1}$, \\ mansur_mg@mail.ru \\ 1 Institute of Geophysics of the Urals Branch of the Russian Academy of Sciences, \\ 100, Amundsen street, Yekaterinburg, 620016, Russia.
}

The relevance of the research. Temperature measurements in boreholes are used for solving a wide range of exploration, geophysical, environmental, hydrogeological, and geodynamic problems. The development of new temperature sensors and registration systems significantly expands the capabilities of borehole thermometry. This raises the requirements for measurement accuracy. However, these requirements often cannot be satisfied in real borehole conditions due to the influence of temperature noise caused by free thermal convection of the fluid. For effective planning of equipment and methods of temperature measurements in boreholes, it is necessary to evaluate the amplitude of temperature noise.

The main aim of the research is to develop mathematical models for estimating the level of temperature noise caused by free thermal convection.

Methods: statistical analysis of temperature records obtained from laboratory experiments, temperature logging and temperature monitoring in boreholes.

Results. The optimal parameter for estimating temperature noise is the standard deviation of temperature fluctuations. Models of varying complexity have been developed that make it possible to estimate the amplitude of convective noise depending on the geothermal gradient, the internal radius of the borehole, and the Rayleigh number. The proposed models allow choosing the equipment and methods of temperature logging and temperature monitoring in boreholes, depending on the ratio of useful signal/temperature noise.

\section{Key words:}

Borehole, temperature logging, temperature monitoring, free thermal convection, temperature noise.

The research was supported by the RFBR, project no. 19-05-00050-a (field research in boreholes, collection and analysis of temperature logging and monitoring data) and state funded topic of $R \& D$ no. 0394-2019-0002 (investigations on the laboratory unit for modeling free thermal convection).

\section{References}

1. Pfister M., Rybach L. High-resolution digital temperature logging in areas with significant convective heat transfer. Geothermics, 1995, vol. 24, pp. 99-100.

2. Wisian K.W., Blackwell D.D., Bellani S., Henfling J.A., Norman R.A., Lysne P.C., Forster A., Schrotter J. Field comparison of conventional and new temperature logging systems. Geothermics, 1998, vol. 27, pp. 131-141.

3. Cermak V., Safanda J., Bodri L. Precise temperature monitoring in boreholes: Evidence for oscillatory convection? P. I. Experiments and field data. International Journal of Earth Sciences, 2008, vol. 97 (2), pp. 365-373.

4. Cermak V., Safanda J., Bodri L. Thermal instability of the fluid column in a borehole: application to the Yaxcopoil hole (Mexico) International Journal of Earth Sciences, 2010, vol. 99 (6), pp. 1437-1451.

5. Berthold S., Börner F. Detection of free vertical convection and double-diffusion in groundwater monitoring wells with geophysical borehole measurements. Environmental geology, 2008 , vol. 54 (7), pp. 1547-1566.

6. Eppelbaum L.V., Kutasov I.M. Estimation of the effect of thermal convection and casing on the temperature regime of boreholes: a review. Journal of Geophysics and Engineering, 2011, vol. 8 (1), pp. 1-10.

7. Demezhko D.Yu., Yurkov A.K., Utkin V.I., Klimshin A.V. On the nature of temperature variations in borehole kun-1 (Kunashir Island). Russian Geology and Geophysics, 2012, vol. 53 (3), pp. 313-319.

8. Valiullin R.A., Sharafutdinov R.F., Fedotov V., Kanafin IV. Experimental device for studying free heat convection during induction heating of the casing. Vestnik Bashkirskogo Universiteta, 2016, vol. 21 (2), pp. 264-268. In Rus.

9. Vélez Márquez M., Raymond J., Blessent D., Philippe M., Simon N., Bour O., Lamarche L. Distributed thermal response tests using a heating cable and fiber optic temperature sensing. Energies, 2018, vol. 11 (11), pp. 1-24.

10. Klepikova M.V., Roques C., Loew S., Selker J. Improved characterization of groundwater flow in heterogeneous aquifers using granular polyacrylamide (PAM) gel as temporary grout. Water Resources Research, 2018, vol. 54 (2), pp. 1410-1419.

11. Diment W.H. Thermal regime of a large diameter borehole: instability of the water column and comparison of air- and waterfilled conditions. Geophysics, 1967, vol. 32, pp. 720-726.

12. Sammel E.A. Convective flow and its effect on temperature logging in small-diameter wells. Geophysics, 1968, vol. 33 (6), pp. 1004-1012

13. Diment W.H., Urban Th.C. A simple method for detecting anomalous fluid motions in boreholes from continuous temperature logs. GRC Trans., 1983, vol. 7, pp. 485-490.

14. Mindubaev M.G., Demezhko D.Yu. Free thermal convection in boreholes: numerical modeling and experimental data. Monitoring. Science and Technologies («MST»), 2012, vol. 4 (13), pp. 12-18. In Rus.

15. Khoroshev A.S. Numerical study of free convective flows in extended vertical cylindrical areas under a constant vertical temperature gradient on the side surface. VESTNIK of Samara University. Aerospace and Mechanical Engineering, 2012, vol. 5-1 (36), pp. 46-48. In Rus. 
16. Demezhko D.Yu., Mindubaev M.G., Khatskevich B.D. Thermal effects of natural convection in boreholes. Russian Geology and Geophysics, 2017, vol. 58 (10), pp. 1270-1276.

17. Khoroshev A.S., Shakhov V.G. The intensity of convection of fluids with different Prandtl number in a vertical cylinder of large aspect ratio. Mathematical Physics and Computer Modeling, 2018, vol. 21 (1), pp. 70-79. In Rus.

18. Demezhko D.Yu., Khatskevich B.D., Mindubaev M.G. Natural Thermal Convection in a Vertical Water-Filled Cylinder: Infrared Thermography Investigation. Russian Geology and Geophysics, 2019, vol. 60(7), pp. 813-818.

19. Kinoshita M., Sayanagi K., Kasaya T., Araki E., Ito H. Long-term temperatures measurements at some onland and sub-seafloor boreholes. JAMSTEC Report of Research and Development, 2009, pp. 89-102.

20. The International Ocean Discovery Program (IODP). Available at: https://www.iodp.org; http://mlp.ldeo.columbia.edu/logdb/ scientific_ocean_drilling/result/ (accessed 15 August 2020).

\section{Information about the authors}

Dmitry Yu. Demezhko, Dr. Sc., chief research scientist, Institute of Geophysics of the Urals Branch of the Russian Academy of Sciences.

Bogdan D. Khatskevich, junior research scientist, Institute of Geophysics of the Urals Branch of the Russian Academy of Sciences.

Mansur G. Mindubaev, Cand. Sc., senior research scientist, Institute of Geophysics of the Urals Branch of the Russian Academy of Sciences.
21. Cuffey K.M., Clow G.D. Temperature profile of the West Antarctic ice sheet divide deep borehole. U.S. Antarctic Program (USAP) Data Center, 2014. Available at: http://www.usapdc.org/view/dataset/609550 (accessed 15 January 2020).

22. Clow G.D., Saltus R.W., Waddington E.D. A new high-precision borehole-temperature logging system used at GISP2, Greenland, and Taylor Dome, Antarctica. Journal of Glaciology, 1996, vol. 42 (142), pp. 576-584.

23. Gershuni G., Zhukhovitskii E. Convective stability of incompressible fluids. Jerusalem, Keter Publ. House, 1976. 336 p.

24. Khatskevich B.D., Demezhko D.Yu., Mindubaev M.G. Sposob temperaturnogo monitoring $v$ vodonapolnennykh skvazhinakh [The way to temperature monitoring in water filled boreholes. Patent RF, no. 2678174, 2019.

Received: 17 June 2020. 